\title{
THE EFFECTIVENESS OF THE MCKENZIE METHOD IN TREATING LUMBAR DISCOPATHY
}

original paper

( ) University School of Physical Education in Wroclaw

DOI: https://doi.org/10.5114/hm.2021.103296

\author{
ARLETTA HAWRYLAK ${ }^{1}$, ANETA DEMIDAS ${ }^{1}$, KRYSTYNA CHROMIK ${ }^{1}$, ADAM HAWRYLAK ${ }^{2}$ \\ ${ }^{1}$ University School of Physical Education in Wrocław, Wrocław, Poland \\ ${ }^{2}$ Physiotherapist; Manus, Długołęka, Poland
}

\begin{abstract}
Purpose. The aim of this study was to evaluate the effectiveness of a 2 -week physiotherapy treatment augmented with the McKenzie method in patients with lumbar discopathy. The influence of this treatment on progression in the mobility range of lumbar spine was investigated.

Methods. Sixty patients aged 40-59 years diagnosed with lumbar discopathy were equally divided into group A (mean age: 51.4 years) and group B (mean age: 51.8 years). The McKenzie method was applied in group A and conservative physiotherapeutic treatment was administered to group B. Pre- and post-intervention measures of spinal range of motion were assessed with a Saunders digital inclinometer; pain intensity and nerve root irritation were also collected. The results were compared between groups.
\end{abstract}

Results. Spinal mobility improved and pain was reduced in both groups albeit enhanced treatment outcomes were observed in group A compared with group B $(p<0.0001)$.

Conclusions. The improved response in group A attests to the applicability of the McKenzie method in the treatment of lumbar discopathy. Post-treatment values did not meet clinical norms and may be a result of group characteristics at baseline (high pain level and impeded spinal mobility) or the limited intervention duration.

Key words: spine, pain, rehabilitation, treatment

\section{Introduction}

Low back pain is one of the most common ailments of the musculoskeletal system. This spinal disorder can negatively affect many activities of daily life and has a significant economic impact in industrialized countries [1, 2]. An epidemiological study finds that $65-80 \%$ of individuals experience low back pain at least once in their lifetime, with $90 \%$ of cases caused by degenerative disc disease [3]. The lumbar spine is particularly predisposed to pathology owing to significant exploitation [4]. McKenzie posits that the onset of chronic pain in this spinal region may be caused by range limitations during extension, in which the nucleus pulposus is shifted forwards and the intradiscal pressure is decreased. Contributing risk factors include work-related mechanical exposure and overuse [5]. Psychogenic factors also play an important role, particularly when pain becomes chronic [6]. Conservative treat- ment for low back pain caused by discopathy is complex as it needs to integrate pharmacotherapy, physiotherapy, and patient education and address the primary event responsible for pain [7]. Of these components, physiotherapy is critical as the underlying basis of physiotherapeutic treatment is to accelerate the reconstructive and recovery processes while preventing the onset of debilitating compensatory strategies $[8,9]$. Clinical evidence supports various treatment modalities designed to reduce increased paraspinal muscle tension, pain, and inflammation. One method that has seen considerable application in Anglo-Saxon countries is the multi-dimensional approach developed by Robin A. McKenzie, in which patients are classified into one of three subgroups, with exercises then tailored to create an individualized rehabilitation protocol [2, 5, 7, 10-12]. The aforementioned literature notes that regular execution can lead to lasting improvements in over $80 \%$ of the patients afflicted with

Correspondence address: Adam Hawrylak, Manus, ul. Klonowa 5, 55-095 Długołęka, Poland,

e-mail: manus-a.h@wp.pl

Received: January 22, 2020

Accepted for publication: October 4, 2020

Citation: Hawrylak A, Demidaś A, Chromik K, Hawrylak A. The effectiveness of the McKenzie method in treating lumbar discopathy. Hum Mov. 2021;22(4):98-104; doi: https://doi.org/10.5114/hm.2021.103296. 
discopathy. A fundamental characteristic of this method is that it involves a large self-treatment component as patients perform exercises at home.

The McKenzie method is commonly administered concomitantly with other standard treatments. These include transcutaneous electrical nerve stimulation, which delivers low frequency pulses with modulated wave patterns in treating acute and chronic pain [13]; laser therapy, which has seen increasing use in treating low back pain and is frequently coupled with electrical nerve stimulation [14]; and pulsed magnetic field therapy, owing to its value in providing analgesic effects, increasing the range of spinal mobility, and enhancing muscle strength for improved static and dynamic balance [15]. In light of the above, the aim of the present study was to evaluate the treatment effects of the McKenzie method compared with standard physiotherapy in a Polish sample of patients with lumbar discopathy. The influence of this treatment on progression in the mobility range of lumbar spine was investigated.

\section{Material and methods}

The participants were randomly divided into 2 subgroups. Group A $(n=30)$ included 15 men and 15 women and group B $(n=30)$ comprised 18 women and 12 men. The anthropometric characteristics of the sample are presented in Table 1.

The study was performed at a specialist orthopaedic rehabilitation health clinic located in Wrocław, Poland. Sixty patients from the clinic were recruited meeting the following inclusion criteria: age of 40-59 years, at least 6 months of chronic back pain, lumbar discopathy diagnosed with X-ray and computerized tomography, no contraindications for participation, and written informed consent. The patients refrained from any pharmacological treatment or other forms of therapy during the study period.

The exclusion criteria were as follows: acute spine pain syndrome in the course of lumbar spine discopathy, cauda equina syndrome, fractures or cancer on the spine, disorder associated with spinal injuries, presence of a 'red flag' criterion, age over 59 or under 40 , lack of the patient's consent to participate in the examination.

In both groups, the treatment was administered daily for 2 weeks, excluding Saturdays and Sundays (10 sessions total). Both groups attended a conservative physiotherapeutic program involving kinesiotherapeutic procedures that also included transcutaneous electrical nerve stimulation, pulsed magnetic field therapy, and laser therapy.

The applied exercises in the field of kinesiotherapy were as follows:

1. Starting position: lying backwards, arms along the body, both lower limbs bent, resting on the mattress. Movement: raise right leg up (knee bent, foot dorsal bend). Keep the final position for 30 seconds. Return to the starting position. Repeat the exercise on the other side.

2. Starting position: lying backwards, torso rested on elbows, both lower limbs bent. Movement: as above.

3. Starting position: half-sitting, hips and knees bent, torso supported on straight elbows. Movement: as above.

4. Starting position: sitting with hips and knees bent, feet on the floor. Movement: as above.

5. Starting position: sitting with hips and knees bent, feet on the floor. Movement: balance sit.

The additional McKenzie-based therapy in group A involved 30 minutes of physiotherapist-supervised exercise with procedures performed when lying prone, prone with extension, prone with extension for long duration, and sitting. The description of the procedures used in accordance with the McKenzie method is the following:

1. Starting position: front lying on a mattress. The upper limbs are arranged along the body. Maintain the position for 5 minutes. Breathe regularly, relax completely, especially reduce the tension in the muscles of the lower lumbar spine, hips, and legs.

2. Starting position: front lying on a mattress. Elbows under the shoulders, bent at a right angle, allowing you

Table 1. Anthropometric characteristics of the study participants

\begin{tabular}{|c|c|c|c|c|c|c|}
\hline & \multirow{2}{*}{\multicolumn{3}{|c|}{ Group A }} & \multirow{2}{*}{\multicolumn{3}{|c|}{ Group B }} \\
\hline & & & & & & \\
\hline & $\bar{x} \pm S D$ & Min & Max & $\bar{x} \pm S D$ & Min & Max \\
\hline Age (years) & $51.4 \pm 3.61$ & 49 & 58 & $51.8 \pm 2.76$ & 48 & 59 \\
\hline Body height (cm) & $164.1 \pm 8.13$ & 156 & 177 & $166.1 \pm 9.16$ & 155 & 180 \\
\hline Body mass (kg) & $75.9 \pm 11.5$ & 59 & 86 & $73.5 \pm 12.5$ & 57 & 84 \\
\hline Body mass index $\left(\mathrm{kg} / \mathrm{m}^{2}\right)$ & $28.2 \pm 2.72$ & 27.9 & 31.2 & $26.6 \pm 2.49$ & 25.5 & 29.1 \\
\hline
\end{tabular}


A. Hawrylak, A. Demidaś, K. Chromik, A. Hawrylak, Effectiveness of the McKenzie method

to lean on your forearms. Maintain the position for 3 minutes. Breathe regularly, relax the muscles of your lower back, hips, and legs. Stay in this position for 2-3 minutes.

3. Starting position: front lying. Elbows bent, palms resting on a mattress at chest level, under the shoulders, as before the push-up exercise. Straightening your elbows, push your upper body upwards as far as the pain allows. Relax your pelvic, hip, and lower limb muscles completely. Also remember to breathe normally, hold this position for a second or two, then lower the body to the starting position; repeat the rises 10 times.

4. Starting position: front lying on the couch. The elbows are bent and hooked on the upper edge of the couch. Then, the subject is gradually lifted by lifting the front part of the bed until it is flexed to an angle of approximately $70^{\circ}$ in the lumbar spine. In this position of extended extension on the couch, stay for 5 minutes.

5. Starting position: sit on a chair with your spine fully extended and with marked lumbar lordosis. Maintain this corrected sitting position for 5 minutes.

Transcutaneous electrical nerve stimulation was administered by using a Multitronic MT-7p electrotherapeutic device. Two standard $6 \times 12$-cm plate electrodes were placed in the lumbar spine area where pain was experienced. The device generated a bidirectional, symmetric, and rectangular impulse. Amperage was individually adjusted, with the average dose of $30 \mathrm{~mA}$. Duration was 20 minutes, with $10-\mathrm{Hz}$ current applied in the first 10 minutes and increased to $100 \mathrm{~Hz}$ in the last 10 minutes of the procedure. Pulsed magnetic field therapy was delivered via a Magnetronic MS-10 device adjusted to 5-7 mT amplitude and 25$30 \mathrm{~Hz}$ frequency. Treatment was directed to the lumbar section for a duration of 20 minutes. Laser therapy involved CTL-1202 and CTL-1106MX scan controllers, calibrated to deliver a dose of $4 \mathrm{~J} / \mathrm{cm}$ in the lumbar area.

Baseline and post-intervention measures were collected in identical conditions for both groups. Anterior and posterior spinal curvatures and lumbar spinal mobility were evaluated with a Saunders digital inclinometer, following the American Medical Association guidelines [16-18]. The patient assumed a standing position (barefoot) with arms resting freely along the trunk. Anatomical landmarks were established at the centre of the sacral bone (point A) and at the intervertebral space between Th12 and L1 (point B). For range of motion of the hip joint, the inclinometer was placed at point $\mathrm{A}$ and reset to zero. The patient then performed a maximum flexion and the value from the inclinometer was recorded. After the participant returned to a neutral standing position, the range of motion of the lumbar spine was assessed by placing the inclinometer at point $B$ and again having the patient perform a maximum flexion. The procedures were then repeated albeit with extension movements instead of flexions. The same landmarks were used and the patient was asked not to shift their hips forward during extension. The results were expressed in degrees and compared with current clinical norms $\left(60^{\circ}\right.$ for flexion and $25^{\circ}$ for extension). Czaprowski et al. [19] recommended that spinal measurements with the Saunderes inclinometer be taken by one examiner to ensure high repeatability, with intra-observer measurement error found to range from $2.8^{\circ}$ to $3.8^{\circ}$.

Low back pain was assessed with a visual analogue scale owing to the high reliability of this method [20]. Patients were asked to quantify the intensity of pain on a 10 -point scale, with 0 representing no pain and 10 standing for agonizing pain. Nerve root irritation was evaluated by using the Straight Leg Raise test. The test is positive for herniated disk (Lasègue's sign) if pain is felt when the lower limb is raised to $80^{\circ}$ flexion $[21,22]$. The procedure involved the patient lying supine on an examination table in a relaxed, unrestrained position. The examiner lifted the straightened leg until sciatic pain was felt. The test was then terminated and the degree of flexion was recorded with a goniometer. The range of motion of the hip joint during flexion was included in the final data set only if the patient tested positive for Lasègue's sign.

Statistical analysis was performed with the Statistica PL 9.0 software (StatSoft) and Microsoft Excel. All data were calculated as means $(\bar{x})$ and standard deviations $(S D)$. The normality of the data set was verified with the Shapiro-Wilk test. Normal distribution was confirmed and intergroup comparisons were performed with Student's $t$-test. The results were considered statistically significant if $p<0.001$.

\section{Ethical approval}

The research related to human use has complied with all the relevant national regulations and institutional policies, has followed the tenets of the Declaration of Helsinki, and has been approved by the Senate Commission for Scientific Research Ethics of the University School of Physical Education in Wrocław, Poland.

\section{Informed consent}

Informed consent has been obtained from all individuals included in this study. 


\section{Results}

Pre-intervention measures show that the range of motion in both groups was severely limited. Spinal mobility during flexion and extension improved in both groups, with an enhanced response observed in group A when compared with group B. This difference was statistically significant (Table 2).

In neither group did the improvement in spinal range of motion meet clinical norms $\left(60^{\circ}\right.$ for flexion and $25^{\circ}$ for extension). The intensity of pain decreased in both groups after the intervention, with the change in group A achieving statistical significance (from 5.61 to 2.65) (Table 3).

Greater improvements in group A were also observed in the Straight Leg Raise test (Table 4). The mean value at which patients in this group tested positive for Lasègue's sign increased from $52.66^{\circ}$ to $71.73^{\circ}$. The post-intervention difference between group $\mathrm{A}$ and group B was statistically significant.

\section{Discussion}

Despite a significant progress in classical and manual therapy, there is a high prevalence of low back pain [2]. The increase in the sedentary lifestyle has further contributed to a reduction in the mobility range of the spine and lowered the resistance of the lumbar spine to static and dynamic load [5, 6]. This warrants the need for more comprehensive preventive and therapeutic methods that address patient education, as well as pharmacologic, physiotherapeutic, and psychotherapeutic treatment $[4,23]$. The efficacy of kinesiotherapy in the treatment of lumbar discopathy is still subject to ongoing debate as to treatment protocols, range, and goals. Generally, this type of treatment administers exercise so as to increase the mobility of the spine, reduce static and dynamic disorders, strengthen the muscle corset, and restore balance in muscle tone $[4,24]$.

Considering the study sample, both groups showed significant limitations in the spinal range of motion prior to the intervention. While significant improvements were observed after treatment, clinical norms were not achieved in either group. This finding suggests that the pre-intervention mobility limitations were severe and that the 2 -week duration of the treatment was insufficient to correct the range of motion. However, the greater improvement observed in group A implies that the application of the McKenzie method may provide an enhanced treatment response [2, 5, 25-30]. This may be due to the fact that standard physiotherapy protocols for low back pain, as administered solely to group B, are designed to eliminate

Table 2. Mean values $(\bar{x})$ and standard deviations $(S D)$ of pre- and post-intervention range of lumbar flexion and extension

\begin{tabular}{|c|c|c|c|c|c|c|c|c|c|}
\hline \multirow{2}{*}{ Group } & \multirow{2}{*}{ Measure } & \multicolumn{4}{|c|}{ Lumbar flexion $\left(^{\circ}\right)$} & \multicolumn{4}{|c|}{ Lumbar extension $\left(^{\circ}\right)$} \\
\hline & & $\bar{x}$ & $S D$ & $t$ & $p$ & $\bar{x}$ & $S D$ & $t$ & $p$ \\
\hline \multirow{2}{*}{$\mathrm{A}$} & Pre-intervention & 11.3 & 11.21 & \multirow{2}{*}{-9.17} & \multirow{2}{*}{$<0.000001^{*}$} & 5.43 & 5.84 & \multirow{2}{*}{-6.24} & \multirow{2}{*}{$<0.000001^{*}$} \\
\hline & Post-intervention & 22.3 & 12.16 & & & 13.36 & 6.11 & & \\
\hline \multirow{2}{*}{ B } & Pre-intervention & 16.8 & 1.38 & \multirow{2}{*}{-8.19} & \multirow{2}{*}{$0.000001^{*}$} & 4.25 & 4.38 & \multirow{2}{*}{-5.19} & \multirow{2}{*}{$0.000001 *$} \\
\hline & Post-intervention & 25.8 & 1.40 & & & 11.25 & 5.40 & & \\
\hline
\end{tabular}

* $p<0.001$

Table 3. Mean values $(\bar{x})$ and standard deviations $(S D)$ of pre- and post-intervention pain intensity as measured with visual analogue scale (VAS)

\begin{tabular}{|c|c|c|c|c|c|}
\hline \multirow{2}{*}{ Group } & \multirow{2}{*}{ Measure } & \multicolumn{4}{|c|}{ VAS pain intensity } \\
\hline & & $\bar{x}$ & $S D$ & $t$ & $p$ \\
\hline \multirow{2}{*}{ A } & Pre-intervention & 5.61 & 1.4 & \multirow{2}{*}{3.5} & \multirow{2}{*}{$<0.000001 *$} \\
\hline & Post-intervention & 2.65 & 1.34 & & \\
\hline \multirow{2}{*}{ B } & Pre-intervention & 4.3 & 1.9 & \multirow{2}{*}{4.3} & \multirow{2}{*}{0.0002} \\
\hline & Post-intervention & 2.9 & 1.7 & & \\
\hline
\end{tabular}

* $p<0.001$
Table 4. Mean values $(\bar{x})$ and standard deviations (SD) of pre- and post-intervention degree of flexion for positive Lasègue's sign

\begin{tabular}{lllll}
\hline \multirow{2}{*}{ Group } & Measure & \multicolumn{3}{c}{ Positive Lasègue's sign $\left(^{\circ}\right)$} \\
\cline { 2 - 4 } & $\bar{x}$ & $S D$ & $t$ & $p$ \\
\hline
\end{tabular}

\begin{tabular}{|c|c|c|c|}
\hline A & $\begin{array}{ll}\text { Pre-intervention } & 52.66 \\
\text { Post-intervention } & 71.73\end{array}$ & $\begin{array}{l}24.41 \\
20.38\end{array}-15.4$ & $<0.000001^{*}$ \\
\hline B & $\begin{array}{ll}\text { Pre-intervention } & 54.3 \\
\text { Post-intervention } & 62.54\end{array}$ & $\begin{array}{l}21.90 \\
19.73\end{array}-14.3$ & 0.00002 \\
\hline
\end{tabular}

${ }^{*} p<0.001$ 
A. Hawrylak, A. Demidaś, K. Chromik, A. Hawrylak, Effectiveness of the McKenzie method

pain [31, 32]. They do not aim to minimize overloadrelated degenerative changes of the intervertebral discs or to halt the posterior shift of the nucleus pulposus. Instead, physiotherapeutic treatment applies methods that facilitate the migration of the nucleus pulposus via antalgic positions by using flexion and similar motor exercises. The rationale for this approach is that flexing the lumbar spine should create an optimal distance between the vertebral arches, unloading the facet joints and widening the intervertebral spaces to release trapped nerve roots and ultimately improve paraspinal tissue function. However, as previously mentioned, such analogical procedures in the treatment of low back pain due to intervertebral disc degeneration do not facilitate the repositioning of the nucleus pulposus. The McKenzie method is credited with treating this condition by the ability to release pressure on nerve roots (as observed in the Straight Leg Raise test) and thus improve the mobility of the lumbar spine [7, 24, 25].

Pain related to lumbar discopathy is caused by irritation of spinal nociceptors or by pressure applied to nerve roots in the spinal canal $[13,15]$. Besides physiotherapeutic methods, additional therapies have been introduced to modulate pain. Transcutaneous electrical nerve stimulation is one of the most common neuromodulation treatments as it shows high efficacy (improvement in $40-60 \%$ of patients), particularly in treating chronic pain [13]. Epidemiological research confirms that the method is effective in reducing pain symptoms in $30 \%$ of cases in which pain does not respond to other forms of analgesic treatment [13]. Other successful methods include the application of magnetic fields and laser therapy for their high analgesic effects [14]. The aforementioned literature suggests that laser therapy is more effective in relieving pain than electrotherapy.

Conservative treatment of low back pain caused by lumbar discopathy should aim to mobilize the patient, restore and increase spinal range of motion, enhance function, and introduce preventive methods that help avoid future episodes [9, 26-30, 33-36]. The literature highlights that patients be made aware of the causes of low back pain and informed about various treatment options and prognoses, as well as future management. This includes information on the risk of recurrence, the importance of performing appropriate exercises to develop a strong muscle corset and maintain healthy body mass, and ways to avoid overuse and improper loading of the spine [4, 9, 23].

The limitations of our research include the selection of the control group: probably, these individuals should not have received any physiotherapy treatment. More precise results would also be achieved if the number of applied physiotherapeutic treatments were limited, both in the field of standard therapy and the McKenzie methods of treatment. Also, tests would need to be performed in a larger population to more accurately define the long-term effects of short-term physiotherapy outcomes.

\section{Conclusions}

Enhanced improvement in the spinal range of motion was observed after physiotherapy augmented with the McKenzie method, thereby suggesting the efficacy of this protocol.

Post-intervention values did not meet threshold norms, implying that the duration of treatment was insufficient or that the patients had severe limitations in spinal range of motion prior to recruitment.

\section{Disclosure statement}

No author has any financial interest or received any financial benefit from this research.

\section{Conflict of interest}

The authors state no conflict of interest.

\section{References}

1. Last AR, Hulbert K. Chronic low back pain: evaluation and management. Am Fam Physician. 2009;79(12): 1067-1074; doi: 10.1080/20786204.2010.10873969.

2. May S, Donelson R. Evidence-informed management of chronic low back pain with the McKenzie method. Spine J. 2008;8(1):134-141; doi: 10.1016/j.spinee.2007. 10.017.

3. Sipko T, Chantsoulis M, Kuczyński M. Postural control in patients with lumbar disc herniation in the early postoperative period. Eur Spine J. 2010;19(3):409-414; doi: 10.1007/s00586-009-1082-x.

4. Hawrylak A, Skolimowski T, Barczyk K, Wójtowicz D. The mobility of the lumbar spine in persons with low back pain. Fizjoter Pol. 2004;4(2):100-106.

5. McKenzie R, May S. The lumbar spine: mechanical diagnosis \& therapy. Waikanae: Spinal Publications; 2003.

6. Adams MA, Mannion AF, Dolan P. Personal risk factors for first-time low back pain. Spine. 1999;24(23):24972505; doi: 10.1097/00007632-199912010-00012.

7. Al-Obaidi SM, Al-Sayegh NA, Nakhi HB, Al-Mandeel M. Evaluation of the McKenzie intervention for chronic low back pain by using selected physical and bio-behavioral outcome measures. PM R. 2011;3(7):637-646; doi: 10.1016/j.pmrj.2011.04.025.

8. Hauggaard A, Persson AL. Specific spinal stabilisation exercises in patients with low back pain - a systematic 
review. Phys Ther Rev. 2007;12(3):233-248; doi: 10.1179/ $108331907 X 222949$.

9. Rasmussen-Barr E, Ang B, Arvidsson I, Nilsson-Wikmar L. Graded exercise for recurrent low-back pain: a randomized, controlled trial with 6-, 12-, and 36-month follow-ups. Spine. 2009;34(3):221-228; doi: 10.1097/ BRS.0b013e318191e7cb.

10. Olczak A, Janiszewski M. The importance of the method of McKenzie in classifying and diagnosing lumbar spine pain syndromes. Med Man. 2002;3(4):26-29.

11. Kilpikoski S, Airaksinen O, Kankaanpää M, Leminen P, Videman T, Alen M. Interexaminer reliability of low back pain assessment using the McKenzie method. Spine. 2002;27(8):E207-E214; doi:10.1097/00007632200204150-00016.

12. Clare HA, Adams R, Maher CG. A systematic review of efficacy of McKenzie therapy for spinal pain. Aust J Physiother. 2004;50(4):209-216; doi: 10.1016/s00049514(14)60110-0.

13. Ratajczak B, Hawrylak A, Demidaś A, Kuciel-Lewandowska J, Boerner E. Effectiveness of diadynamic currents and transcutaneous electrical nerve stimulation in disc disease lumbar part of spine. J Back Musculoskelet Rehabil. 2011;24(3):155-159; doi: 10.3233/ BMR-2011-0289.

14. Ratajczak B, Ryfa R, Boerner E, Kuciel-Lewandowska J, Hawrylak A, Demidaś A. Assessment the influence of the lasertherapy and magnetotherapy in connection with kinesitherapy used by patients with the degenerative low back disease [in Polish]. Adv Rehab. 2011;25(2):13-18; doi: 10.2478/rehab-2013-0005.

15. Melzack R. From the gate to the neuromatrix. Pain. 1999; Suppl 6:S121-S126; doi: 10.1016/S0304-3959(99)00 145-1.

16. Saunders HD. Saunders digital inclinometer. Chaska: Saunders Group Inc.; 1998.

17. American Medical Association. AMA guides to the evaluation of permanent impairment, $4^{\text {th }}$ ed. Chicago: AMA; 1993.

18. Hawrylak A, Chromik K, Ratajczak B, Barczyk-Pawelec K, Demczuk-Włodarczyk E. Spinal range of motion and plantar pressure in sport climbers. Acta Bioeng Biomech. 2017;19(2):169-173; doi: 10.5277/ABB-006472016-01.

19. Czaprowski D, Pawłowska P, Gębicka A, Sitarski D, Kotwicki T. Intra- and interobserver repeatability of the assessment of anteroposterior curvatures of the spine using Saunders digital inclinometer. Ortop Traumatol Rehabil.2012;14(2):145-153; doi: 10.5604/15093492. 992283.

20. Price DD, McGrath PA, Buckingham B. The validation of visual analogue scales as ratio scale measures for chronic and experimental pain. Pain. 1983;17(1):45-56; doi: 10.1016/0304-3959(83)90126-4.

21. Capra F, Vanti C, Donati R, Tombetti S, O’Reilly C, Pillastrini $\mathrm{P}$. Validity of the straight-leg raise test for pa- tients with sciatic pain with or without lumbar pain using magnetic resonance imaging results as a reference standard. J Manipulative Physiol Ther. 2011;34(4): 231-238; doi: 10.1016/j.jmpt.2011.04.010.

22. Majlesi J, Togay H, Unalan H, Toprak S. The sensitivity and specificity of the Slump and the Straight Leg Raising tests in patients with lumbar disc herniation. J Clin Rheumatol. 2008;14(2):87-91; doi: 10.1097/RHU.0b $013 \mathrm{e} 31816 \mathrm{~b} 2 \mathrm{f} 99$.

23. Chou R, Huffman LH, American Pain Society, American College of Physicians. Nonpharmacologic therapies for acute and chronic low back pain: a review of the evidence for an American Pain Society. American College of Physicians clinical practice guideline. Ann Intern Med. 2007;147(7):492-504; doi: 10.7326/0003-4819147-7-200710020-00007.

24. Slade SC, Keating JL. Measurement of participant experience and satisfaction of exercise programs for low back pain: a structured literature review. Pain Med. 2010;11(10):1489-1499;doi:10.1111/j.1526-4637.2010. 00951.x.

25. Petersen T, Larsen K, Nordsteen J, Olsen S, Fournier G, Jacobsen S. The McKenzie method compared with manipulation when used adjunctive to information and advice in low back pain patients presenting with centralization or peripheralization: a randomized controlled trial. Spine. 2011;36(24):1999-2010; doi: 10.1097/BRS. 0b013e318201ee8e.

26. Lam OT, Strenger DM, Chan-Fee M, Pham PT, Preuss RA, Robbins SM. Effectiveness of the McKenzie method of mechanical diagnosis and therapy for treating low back pain: literature review with meta-analysis. J Orthop Sports Phys Ther. 2018;48(6):476-490; doi: 10.2519/ jospt.2018.7562.

27. Namnaqani FI, Mashabi AS, Yaseen KM, Alshehri MA. The effectiveness of McKenzie method compared to manual therapy for treating chronic low back pain: a systematic review. J Musculoskelet Neuronal Interact. 2019;19(4):492-499.

28. Szulc P, Wendt M, Waszak M, Tomczak M, Cieślik K, Trzaska T. Impact of McKenzie method therapy enriched by muscular energy techniques on subjective and objective parameters related to spine function in patients with chronic low back pain. Med Sci Monit. 2015;21:29182932; doi: 10.12659/MSM.894261.

29. Garcia AN, Menezes Costa LC, Mota da Silva T, Barreto Gondo FL, Cyrillo FN, Costa RA, et al. Effectiveness of back school versus McKenzie exercises in patients with chronic nonspecific low back pain: a randomized controlled trial. Phys Ther. 2013;93(6):729-747; doi: 10.2522/ ptj.20120414.

30. Halliday MH, Pappas E, Hancock MJ, Clare HA, Pinto RZ, Robertson G, et al. A randomized controlled trial comparing the McKenzie method to motor control exercises in people with chronic low back pain and a directional preference. J Orthop Sports Phys Ther. 2016; 46(7):514-522; doi: 10.2519/jospt.2016.6379. 


\section{HUMAN MOVEMENT}

A. Hawrylak, A. Demidaś, K. Chromik, A. Hawrylak, Effectiveness of the McKenzie method

31. Rajfur J, Pasternok M, Rajfur K, Walewicz K, Fras B, Bolach B, et al. Efficacy of selected electrical therapies on chronic low back pain: a comparative clinical pilot study. Med Sci Monit. 2017;23:85-100; doi: 10.12659/ msm.899461.

32. De Almeida CC, da Silva VZM, Cipriano G Júnior, Liebano RE, Durigan JLQ. Transcutaneous electrical nerve stimulation and interferential current demonstrate similar effects in relieving acute and chronic pain: a systematic review with meta-analysis. Braz J Phys Ther. 2018;22(5):347-354; doi: 10.1016/j.bjpt.2017.12.005.

33. Bronfort G, Haas M, Evans RL, Bouter LM. Efficacy of spinal manipulation and mobilization for low back pain and neck pain: a systematic review and best evidence synthesis. Spine J. 2004;4(3):335-356; doi: 10.1016/j. spinee.2003.06.002.

34. Slade SC, Keating JL. Unloaded movement facilitation exercise compared to no exercise or alternative therapy on outcomes for people with nonspecific chronic low back pain: a systematic review. J Manipulative Physiol Ther. 2007;30(4):301-311; doi: 10.1016/j.jmpt.2007. 03.010 .

35. Saur PM, Ensink FB, Frese K, Seeger D, Hildebrandt J. Lumbar range of motion: reliability and validity of the inclinometer technique in the clinical measurement of trunk flexibility. Spine. 1996;21(11):1332-1338; doi: 10.1097/00007632-199606010-00011.

36. Broetz D, Hahn U, Maschke E, Wick W, Kueker W, Weller M. Lumbar disk prolapse: response to mechanical physiotherapy in the absence of changes in magnetic resonance imaging. Report of 11 cases. NeuroRehabilitation. 2008;23(3):289-294; doi: 10.3233/NRE-200823312. 